\title{
Qualidade microbiológica de cortes cárneos utilizados para elaboração de carne de sol no Norte de Minas Gerais submetidos a diferentes tecnologias de conservação*
}

\author{
Microbiological quality of beef cuts used in the preparation of sun- \\ dried meat in the North of Minas Gerais, Brazil, submitted to different \\ conservation technologies
}

\author{
Daniela Ribeiro de Souza, ${ }^{* *}$ Anna Christina de Almeida, ${ }^{* * *}$ Rogério Marcos de Souza, ${ }^{* * *}$ Camila Almeida de Jesus, ${ }^{* *}$
} Fernanda Santos Silva Raidan ${ }^{* * * *}$

\begin{abstract}
Resumo
Carne de sol é um produto obtido pela salga leve e discreta e posterior desidratação pelo ar atmosférico dos cortes cárneos de bovinos, sendo um produto muito apreciado no Norte de Minas Gerais. A falta de regulamentação técnica e utilização de tecnologia adequada para o processamento e armazenamento contribuem para baixa qualidade sanitária e coloca em risco a saúde do consumidor. O objetivo deste trabalho foi avaliar e comparar a contaminação microbiológica entre amostras de carne bovina - chã de fora (coxão duro), utilizadas no preparo da carne de sol, em diferentes locais e condições de armazenamento. Foram analisadas 15 amostras. Os resultados obtidos na pesquisa de coliformes variaram de $<3,0 \mathrm{NMP} / \mathrm{g}$ a $>1100 \mathrm{NMP} / \mathrm{g}$. Foram constatadas contagens de Staphylococcus spp., variando de 5,5 × $10^{3}$ a 2,9 $\times 10^{6} \mathrm{UFC} / \mathrm{g}$. A presença de Escherichia coli foi confirmada em $66,67 \%$ e de Staphylococcus coagulase positiva em $26,67 \%$ das amostras analisadas. O produto não está adequado para o consumo, pois apresentou elevada contagem de micro-organismos patogênicos capazes de causar surtos alimentares, além da possibilidade de comprometer a qualidade do produto final.
\end{abstract}

Palavras-chave: armazenamento, contaminação, micro-organismos.

\begin{abstract}
Sun-dried meat is a product obtained through slight salted and dehydrated by the atmospheric air of cuts of beef, which is a product much appreciated in the North of Minas Gerais. Deficient technical regulations and use of appropriate technology for processing and storage contribute to a low health quality and endangers the health of the consumer. The aim of this study was to evaluate and compare the microbiological contamination between samples of beef - Biceps femoris, used in the preparation of sun-dried meat, in different places and storage conditions. 15 samples were analyzed. The results of coliforms obtained in the study ranged from < 3.0 NMP/g to > $1100 \mathrm{NMP} / \mathrm{g}$. Staphylococcusspp counts ranged from $5.5 \times 103$ to $2.9 \times 106 \mathrm{UFC} / \mathrm{g}$. The presence of Escherichia coli was confirmed in $66.67 \%$ and Staphylococcus positive coagulase in $26.67 \%$ of the samples analyzed. The product is not appropriate for consumption because it showed high counts of pathogenic microorganisms sufficient to cause foodborne outbreaks, furthermore the possibility of compromising the quality of final product.
\end{abstract}

Keywords: contamination, microorganisms, storage.

\section{Introdução}

Carne de sol é um produto obtido pela salga leve e discreta e posterior desidratação pelo ar atmosférico dos cortes cárneos de bovinos (Nóbrega, 1982). É um produto muito apreciado no Norte de Minas Gerais, tradicional na culinária da região promovendo o desenvolvimento da pecuária e do comércio (Nobre, 2009).

A carne de sol surgiu no Nordeste, como alternativa para preservar a produção excedente de carne bovina devido às dificuldades encontradas na sua conservação e ao baixo nível econômico da população que não dispunha de equipamentos para refrigeração. Dessa forma, a salga e a desidratação tornaram-se processos de conservação usuais (Mennucciet al., 2010). A utilização destas técnicas, na produção artesanal acabou popularizando este alimento, sendo, geralmente, obtido em condições higiênico-sanitárias insatisfatórias, resultando na diminuindo da vida-de-prateleira desse produto (Costa e Silva, 2001).

O produto passou a ser consumido regionalmente nos centros urbanos, como São Paulo e Rio de Janeiro na elaboração de algumas receitas e diversas preparações culinárias. Devido às suas características de conveniência, esse produto tem grande

*Recebido em 29 de junho de 2014 e aceito em 20 de setembro de 2015.

**Instituto de Ciências Agrárias da Universidade Federal de Minas Gerais. Acadêmica do Curso de Graduação em Engenharia de Alimentos.

***Docente do Instituto de Ciências Agrárias da Universidade Federal de Minas Gerais. Av. Universitária, 1000, Bairro Universitário, Cep: 39404-547.Montes Claros, MG. Email: aca2006@ica.ufmg.br.

****Escola de Veterinaria da Universidade Federal de Minas Gerais. Doutoranda em Zootecnia. 
potencial para conquistar o consumidor doméstico, que busca diversidade de opções de carne de preparo rápido (Mennucciet al., 2010).

A carne de sol não possui normas técnicas para sua produção e comercialização nem padrão de identidade e qualidade oficial estabelecida pelo Regulamento de Inspeção Industrial e Sanitária de Produtos de Origem Animal (Brasil, 1997). A elaboração desse produto segue conceitos ou normas típicas ou regionais em condições precárias de higiene e sem controle de qualidade, podendo apresentar risco à saúde do consumidor (Cruz, 2010).

A microbiota da carne depende das condições nas quais os animais foram criados, abatidos e processados (Costa e Silva, 2001). Os micro-organismos Staphylococcus spp. e Escherichia coli são indicadores de qualidade sanitária, identificando falhas na manipulação dos alimentos (Miranda et al., 2012). O crescimento dessas bactérias potencialmente patogênicas pode ser inibido pelas condições de estocagem e, sobretudo, pela redução da temperatura, pela salga, desidratação (Oliveira et al., 2008).

Devido à crescente comercialização, há necessidade da definição de critérios e padrões físico-químicos para sua elaboração, pois este produto parcialmente desidratado e semipreservado pela salga não pode se enquadrar no padrão existente para o charque e jerked beef, porque sua vida-deprateleira é muito curta quando comparada a estes produtos (Costa e Silva, 2001).

Dessa forma, objetivou-se avaliar e comparar a contaminação microbiológica entre amostras de carne bovina, utilizadas no preparo da carne de sol, em diferentes locais e condições de armazenamento.

\section{Material e métodos}

As amostras de carne bovina - chã de fora (coxão duro), utilizadas no preparo da carne de sol foram selecionados de forma aleatória em 15 estabelecimentos na cidade de Montes Claros, Minas Gerais, Brasil sendo 5 açougues em um bairro, 5 supermercados e 5 boxes de comercialização de carne no Mercado Municipal de Montes Claros/MG, coletando-se1amostra de carne de sol em cada local, totalizando 15 amostras. Estas foram identificadas e transportadas até o laboratório de Microbiologia Aplicada da Universidade Federal de Minas Gerais, Campus Montes Claros, em caixas isotérmicas.

Cada amostra possuía em média $75 \mathrm{~g}$ e obtiveram-se 3 subamostras de 25 gramas, as quais foram denominadas A, B, C. A amostra A foi analisada imediatamente (amostra controle), a amostra $B$ foi embalada a vácuo e acondicionada na geladeira (amostra refrigerada), a $2^{\circ} \mathrm{C}$ e a amostra $\mathrm{C}$ acondicionada no congelador (amostra congelada), a $-18^{\circ} \mathrm{C}$ permanecendo 35 dias e posteriormente realizadas as análises. O período de 35 dias foi definido para análises, considerando-se o prazo de validade encontrado nas embalagens de carne de sol refrigerada disponibilizados no comércio na região de estudo.

As amostras foram submetidas às seguintes análises: determinação do Número Mais Provável (NMP) de coliformes totais, pesquisa de Escherichia colie contagem de Staphylococcus spp. e pesquisa de Staphylococcus coagulase positiva. Essas análises foram realizadas de acordo com a metodologia descrita no American Public Health Association (APHA, 2001).

Os resultados obtidos nas análises microbiológicas foram submetidos ao teste Kruskal-Wallis para comparação entre os tratamentos e entre os locais com nível de significância de 5\%. Para verificar a distinção entre as médias, aplicou-se o teste de Comparações Múltiplas de Dunn. O software utilizando foi o SAS (StatisticalAnalysis System) para Windows versão 8.0.

\section{Resultados e discussão}

As médias de resultados obtidos nas análises microbiológicas estão apresentadas nas Tabelas 1 e 2.

Tabela 1: Média da contagem (Log NMP/g) de coliformes totais das amostras de carne bovina analisadas

\begin{tabular}{cccc}
\hline \multirow{2}{*}{ Tratamentos } & \multicolumn{3}{c}{$\begin{array}{c}\text { Média da contagem (Log NMP/g) } \\
\text { de coliformes totais }\end{array}$} \\
\cline { 2 - 4 } & Mercado & Supermercado & Açougue \\
\hline Controle & $2.05^{\mathrm{Aa}}$ & $2.05^{\mathrm{Aa}}$ & $1.70^{\mathrm{Aa}}$ \\
Refrigerado & $1.99^{\mathrm{Aa}}$ & $2.05^{\mathrm{Aa}}$ & $1.53^{\mathrm{Aa}}$ \\
Congelado & $2.54^{\mathrm{Aa}}$ & $1.66^{\mathrm{ABa}}$ & $1.14^{\mathrm{Ba}}$ \\
\hline
\end{tabular}

*Médias seguidas da mesma letra maiúscula, entre linhas, não diferem significativamente $(p<0,05)$ para os diferentes locais de coleta; médias seguidas da mesma letra minúscula, entre colunas, não diferem significativamente $(p<0,05)$ para os tipos de tratamento.

Para todos os locais onde a carne foi coletada não houve redução da contagem de coliformes totais nas análises realizadas imediatamente após a coleta (controle) ou após o resfriamento e congelamento. Esse resultado era esperado visto que a redução da temperatura não elimina contaminações anteriores. Esse método auxilia o processo de conservação inibindo o desenvolvimento da população de micro-organismos já existente (Alves et al., 2010; Cordeiro et al., 2007).

Para amostras congeladas foi observada menor contagem de coliformes naquelas coletadas nos açougues e maior no Mercado Municipal. Essa diferença pode estar relacionada com a deficiência na metodologia do número mais provável (NMP), para a quantificação de coliformes (McCarthy et al., 1958).

Os resultados obtidos nessa pesquisa de coliformes totais variaram de $<3,0 \mathrm{NMP} / \mathrm{g}$ a $>1100 \mathrm{NMP} / \mathrm{g}$. Entretanto, o resultado obtido na pesquisa de coliformes totais realizada por Oliveira

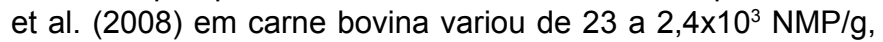
$1,4 \times 10^{3} \mathrm{NMP} / \mathrm{g}$, desvio padrão $1,0 \times 10^{3} \mathrm{NMP} / \mathrm{g}$, sendo que $20 \%$ das amostras analisadas apresentaram-se entre 10 e $10^{2}$ $\mathrm{NMP} / \mathrm{g}, 10 \%$ entre $10^{2}$ e $10^{3} \mathrm{NMP} / \mathrm{g}$ e $70 \%$ das amostras entre $10^{3}$ e $10^{4} \mathrm{NMP} / \mathrm{g}$.

A Resolução RDC n¹26 não dispõe de padrões microbiológicos para coliformes em carne bovina (Brasil, 2001). As bactérias do grupo coliformes são utilizadas como indicadoras das condições higiênico-sanitárias, na avaliação da qualidade de alimentos como a carne, devido à facilidade de seu isolamento, identificação, e uma maior rapidez no diagnóstico final (Oliveira et al., 2008). A presença de coliformes na carne bovina indica contaminação durante o abate, manipulação, como também 
contaminação fecal, podendo haver presença de microorganismos patogênicos no produto (Costa e Silva, 2001).

A Tabela 2 apresenta os resultados da contagem de Staphylococcus spp. Para amostras de carne coletadas no Mercado Municipal e supermercados não houve diferença significativa $(p>0,05)$ para análises de Staphylococcus spp. realizadas imediatamente após a coleta (controle), após o resfriamento ou congelamento (Tabela 2). No entanto, observouse que todos os tipos de tratamentos apresentaram diferença significativa, com a média de contagem de Staphylococcus spp. menor $(p<0,05)$ nas amostras provenientes do açougues.

Tabela 2: Média da contagem (Log UFC/g) de Staphylococcus spp. das amostras de carne bovina analisadas

\begin{tabular}{cccc}
\hline \multirow{2}{*}{ Tratamentos } & \multicolumn{3}{c}{ Média da contagem (Log UFC/g) de } \\
& Staphylococcus spp. \\
\cline { 2 - 4 } & Mercado & Supermercado & Açougue \\
\hline Controle & $4.96^{\mathrm{Aa}}$ & $6.31^{\mathrm{Ba}}$ & $6.35^{\mathrm{Ba}}$ \\
Refrigerado & $5.04^{\mathrm{Aa}}$ & $6.28^{\mathrm{Ba}}$ & $5.41^{\mathrm{ABb}}$ \\
Congelado & $4.79^{\mathrm{Aa}}$ & $6.21^{\mathrm{Ba}}$ & $4.59^{\mathrm{Ac}}$ \\
\hline
\end{tabular}

*Médias seguidas da mesma letra maiúscula, entre linhas, não diferem significativamente $(p<0,05)$ para os diferentes locais de coleta; médias seguidas da mesma letra minúscula, entre colunas, não diferem significativamente $(p<0,05)$ para os tipos de tratamento.

Com relação aos locais, houve diferença significativa $(p<0.05)$ para todos os tipos de tratamentos. Na Tabela 2, observa-se que as amostras de carne bovina analisadas com as condições do tipo controle e refrigerado obtiveram menor média de contagem para Staphylococus spp. no mercado. No estudo realizado por Costa e Silva (2001) a média de contagem de Staphylococcus spp. foi mais elevada para os estabelecimentos não inspecionados que comercializavam carnes.

Uma explicação para esse fato, é que no mercado existe um menor tempo de estocagem da carne, resultando em um menor desenvolvimento microbiano, ou ainda a possibilidade de contaminação da carne no supermercado pela falta de higiene dos utensílios e através do manipulador. A contaminação da carne de sol por Staphylococcus spp., provavelmente, está relacionada com a manipulação da carne pelos vendedores, visto que esses micro-organismos estão presentes naturalmente nas fossas nasais e na pele dos seres humanos (Cruz, 2010).

Mesmo após o processo de refrigeração e congelamento é possível encontrar as mesmas contagens microbiológicas antes da aplicação dessas tecnologias, pois esse processo não apresenta ação esterilizante, apenas retarda a velocidade das atividades microbianas já existentes na carne e impede o surgimento de novos agentes deteriorantes (Alves et al., 2010; Cordeiro et al., 2007).

O gênero de bactérias, Staphylococcus spp. são halotolerantes, crescem bem em até $15 \%$ de sal. A carne de sol é curada exclusivamente pela adição de relativamente baixa concentração de cloreto de sódio, em média 5,0\% oscilando entre 2,9 e 11,9\% Costa e Silva (2001). A matéria-prima, cortes de carnes bovinos, analisadas é utilizada na produção da carne de sol, na qual é adicionado sal para conservação. Portanto, mesmo após a utilização desse método de conservação, o cloreto de sódio não é suficiente para eliminar esse gênero da carne de sol. A presença de Staphylococcus spp. também pode ser utilizada como indicativo de condições inadequadas de manipulação.

A presença de Escherichia coli foi verificada em $66,67 \%$ das amostras analisadas. $O$ percentual de amostras de carne com presença confirmada de Eschericha coli (Figura 1) mostra que a embalagem a vácuo, resfriamento e congelamento tiveram um efeito positivo para a redução deste micro-organismo. $\mathrm{Na}$ pesquisa realizado por Campos et al. (1999), das 17 amostras de carne bovina analisadas, $47,1 \%$ de coliformes totais apresentaram resultados acima de $10^{2} \mathrm{NMP} / \mathrm{g}$; em $35,6 \%$ foi detectada a presença de coliformes fecais e em $17,6 \%$ confirmou-se a presença de Escherichia coli.

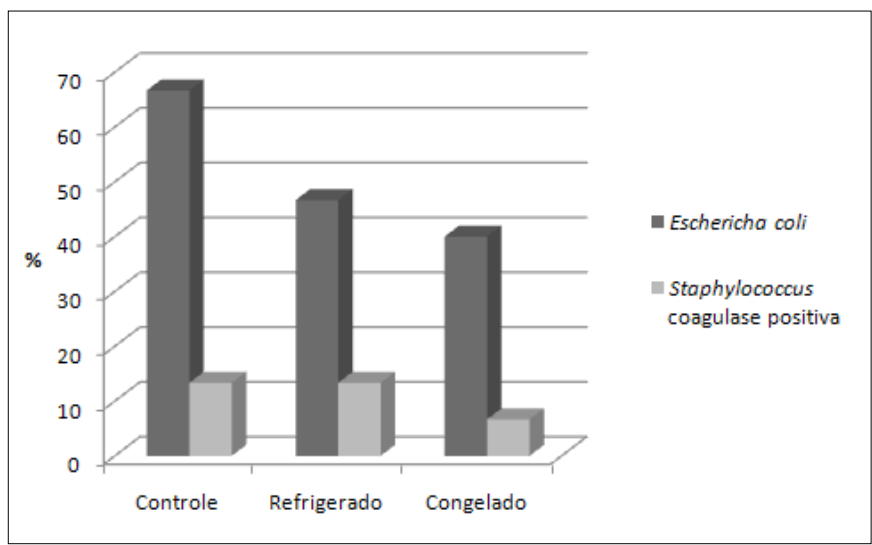

Figura 1: Percentual de amostras de carne de sol com presença das bactérias Escherichia colie Staphylococcus coagulase positiva nos diferentes tratamentos.

A legislação brasileira não dispõe de padrões para coliformes totais, termotolerantes e Escherichia coli, nem a Resolução RDC n`12 estabelece padrões para estes micro-organismo na carne bovina (Brasil, 2001). Segundo Costa e Silva (2001) a presença desta bactéria não é tolerada, mesmo em pequenas quantidades, visto que algumas cepas são comprovadamente enterotoxigênicas e têm sido envolvidas em surtos de gastroenterites severas.

O armazenamento da carne bovina sob temperaturas de refrigeração adequada, e com embalagem a vácuo é importante para reduzir o crescimento de micro-organismos patogênicos e deteriorantes, contribuindo para o prolongamento da vida de prateleira, mas não é possível a eliminação dos microorganismos apenas com a diminuição da temperatura (Carvalho Júnior, 2002; Lima et al. 2011; Costa, 2001).

A incidência de Staphylococcus coagulase positiva foi confirmada em $26,67 \%$ das amostras analisadas. Staphylococcus coagulase positiva reduziu o percentual de incidência (Figura 1), após a análise microbiológica das amostras mantidas sob congelamento. Mesmo com moderada presença de Staphylococcus coagulase positiva, na maioria das amostras contagem foi superior a $5 \mathrm{Log}$ UFC/g.

Staphylococcus coagulase positiva é um agente patogênico que produz enterotoxina, na qual é termoestável e está presente no alimento mesmo após o cozimento, possibilitando, desta forma, a instalação de um quadro de intoxicação de origem alimentar (Cunha Neto et al., 2002) Contagens superior a 5 log UFC/g desse micro-organismo representa risco da presença 
da enterotoxina suficiente para provocar intoxicação alimentar (Costa e Silva, 2001).

O resultado da pesquisa de Staphylococcus coagulase positiva por Campos et al. (1999) foi <100 UFC/g em todas as amostras analisadas. Já Oliveira et al. (2002) obtiveram resultados positivos em $33 \%$ das amostras analisadas de carne bovina, resultado semelhante ao encontrado neste estudo.

A porcentagem de amostras de carne bovina com presença de Eschericha coli e Staphylococcus coagulase positiva em diferentes locais então apresentadas na Figura 2.

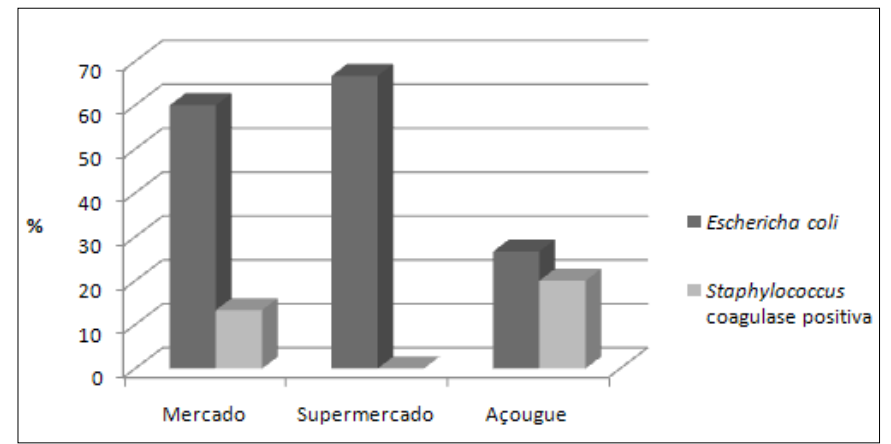

Figura 2: Percentual de amostras de carne de sol com presença de microorganismos - as bactérias Escherichia coli e Staphylococcus coagulase positiva nos diferentes locais de comercialização.

A carne de sol comercializada nos supermercados geralmente é mantida sob refrigeração com a temperatura variando de $7^{\circ} \mathrm{C}$ a $15^{\circ} \mathrm{C}$; nos açougues dos bairros da cidade e nos boxes do mercado municipal a carne é vendida pendurada em ganchos ou sobre o balcão, às vezes armazenada sob refrigeração apenas no período noturno (Miranda e Barreto. 2012).

O percentual de Staphylococcus coagulase positiva foram maior no açougue (20\%), seguido pelo mercado $(13,33 \%)$ e não apresentou incidência no supermercado (0\%) (Figura 2).

Na Figura 2 observa-se que as amostras com maior incidência de $E$. coli foram, por ordem decrescente, no supermercado $(66,67 \%)$, mercado $(60 \%)$ e açougue $(26,67 \%)$. Apesar do emprego do vácuo e congelamento retardar o crescimento microbiológico e aumentar a vida de prateleira dos produtos cárneos, a incidência desse micro-organismo no estabelecimento que emprega essas tecnologias pode ocorrer devido a falhas na higienização pessoal, dos utensílios, na manipulação da matéria-prima e do produto final.

\section{Conclusão}

O produto não esta adequado para o consumo, pois apresentou elevada contagem de micro-organismos patogênicos capazes de causar surtos alimentares. O acondicionamento do produto sob refrigeração e congelamento é importante para retardar o crescimento dos micro-organismos e aumentar a vida de prateleira, mas não elimina a contaminação já existente nas amostras. Por isso é necessário atentar-se para as condições higiênico-sanitárias, evitando contaminações durante a manipulação da carne de sol.

\section{Agradecimentos}

À FAPEMIG - PROCESSO CVZ - APQ-02900-13, PBEXT/UFMG, PROGRAD/PRO-NOTURNO UFMG, PRPq/UFMG.

\section{Referências}

ALVES, L.L.; DELBEM, Á.C.B.; ABREU, U.G.P.; LARA, J.A.F. Avaliação físico-química e microbiológica da carne soleada do Pantanal. Ciência e Tecnologia de Alimentos, v. 30, n. 3, p. 729734, 2010.

AMERICAN PUBLIC HEALTH ASSOCIATION - APHA. Compendium of methods for the microbiological examination of foods.4.ed. Washington:APHA, 2001. 676 p.

AZEVEDO, A.R.P.; MORAIS. T.V.M. A tecnologia da produção da carne-de-sol e suas implicações nos aspectos higiênicossanitários. Revista Nacional da Carne, São Paulo, n. 336, p. 36-50, 2005.

BRASIL. Ministério da Agricultura. Departamento Nacional de Inspeção de Produtos de Origem Animal. Regulamento da Inspeção Industrial e Sanitária de Produtos de Origem Animal R.I.I.S.P.O.A. Aprovado pelo decreto n. 30691 de 29 de março de 1952, alterado pelo Decreto 1255 de 25de junho de 1962. Alterado pelo Decreto 2244 de 04/06/1997. Brasília, DF,1997.

BRASIL. Agência Nacional de Vigilância Sanitária. Resolução ${ }^{\circ}$. 12 de 2 de janeiro de 2001. Regulamento Técnico sobre Padrões Microbiológicos para Alimentos. Diário Oficial da União. Brasília, DF, 12 jan. 2001.

CAMPOS, M.R.H. et al. Estudo das condições microbiológicas no fluxograma de preparação de carne bovina do cardápio de um serviço de alimentação, na cidade de Goiânia-GO. Revista Higiene Alimentar, v.13, p. 66-67, 1999.
CARVALHO JUNIOR, B.C. Estudo da evolução das carnes bovinas salgadas no Brasil e desenvolvimento de um produto de conveniência similar à carne-de-sol. 2002. s.n. Tese (Doutorado) - Universidade Estadual de Campinas. Campinas - SP. 2002.

CORDEIRO, D.; LOPES, T.G.G.; OETTERER, M.; PORTO, E.; GALVÃO, J.A. Qualidade do mexilhão Perna perna submetido ao processo combinado de cocção, congelamento e armazenamento. Boletim do Centro de Pesquisa de Processamento de Alimentos, v. 25, n. 1, p. 165-179, 2007.

COSTA E.L.; SILVA J.A. Avaliação Microbiológica da carne de sol elaborada com baixos teores de cloreto de sódio. Ciência e Tecnologia de Alimentos, v. 21, n. 2, p. 149-153, 2001.

COSTA E.L.; SILVA J.A. Qualidade sanitária da carne de sol comercializada em açougues e supermercados de João Pessoa - PB. Boletim do Centro de Pesquisa de Processamento de Alimentos, v.17 n. 2, p.137-44, 1999.

CRUZ, A.L.M. Produção, comercialização, consumo, qualidade microbiológica e características físico-químicas da carne de sol do Norte de Minas Gerais. 2010. 95 f. Dissertação (Mestrado em Ciências Agrárias) - Instituto de Ciências Agrárias da Universidade Federal de Minas Gerais, Montes Claros, 2010.

CUNHA NETO, A.; SILVA, C.G.M.; STAMFORD, T.L.M. Staphylococcus enterotoxigênicos em alimentos in natura e processados no estado de Pernambuco, Brasil. Ciência e Tecnologia de Alimentos, v. 22, n. 3, p. 263-271, 2002.

FORNIAS,O.V.; DÍAZ, C.V. Clasificación de los Productos Cárnicos. Revista Cubana de Alimentación y Nutrición, v. 13, n. 1, p. 63-67, 1999. 
LIMA, C.M.F. Monitoramento das temperaturas de equipamentos de refrigeração em supermercados da cidade de Maceió, Al. Revista Higiene Alimentar, v. 25, n. 194/195, p. 35-39, 2011.

MCCARTHY, J.A.; THOMAS, H.A.; DELANEY, J.E. Evaluation of the reliability of coliform density tests. American Journal of Public Health and the Nations Health, v. 48, n. 12, p. 1628-1635, 1958.

MIRANDA, P.C.; CAZETTA, M.L.; BARRETO, N.S.E. Carne de sol bovina: Aspectos higiênico-sanitários. Revista Higiene Alimentar, v. 26, n. 214/215, p. 65-68, 2012.

MIRANDA, P.C.; BARRETO, N.S.E. Avaliação higiênico-sanitária de diferentes estabelecimentos de comercialização da carne-desol no município de Cruz das Almas-BA. Revista Caatinga, v. 25 , n. 2, p. 166-172, 2012.

MENNUCCI T.A.; MARCIANO M.A.M.; ATUI M.B.; POLI NETO A.; GERMANO P.M.L. Avaliação da contaminação por matérias estranhas em carne de sol comercializada em "casas do norte". Revista do Instituto Adolfo Lutz, v. 69, n. 1, p. 47-54, 2010.
NOBRE, G.M.C.R. Caracterização físico-química e microbiológica da carne de sol serenada e dos estabelecimentos produtores de um município do norte de Minas Gerais. 2009. 90 f. Dissertação (Mestrado em Ciência e Tecnologia de Alimentos) - Centro Universitário de Belo Horizonte,UNI-BH, Belo Horizonte, 2009.

NÓBREGA, D.M. Contribuição ao estudo da carne de sol visando melhorar sua conservação. Campinas, 1982. 81 p. Tese (Mestrado em Tecnologia de Alimentos), Faculdade de Engenharia de Alimentos - Universidade Estadual de Campinas/ UNICAMP. 1982.

OLIVEIRA, N.M.S.; NASCIMENTO, L.C.; FIORINI, J.E. Isolamento e identificação de bactérias facultativas mesófilas em carnes frescas bovinas e suínas. Revista Higiene Alimentar, v. 16, n. 94, p. 68-74, 2002.

OLIVEIRA, S.; SILVA, J.A.; MACIEL, J.F; AQUINO, J. Avaliação das condições sanitárias de carne bovina comercializada em supermercados de João Pessoa. Alimentos e Nutrição Araraquara, v.19, n. 1, p. 61-66, 2008. 